\title{
Research Paper: Comparing Symptoms of Post-Polio Syndrome in Athlete and Non-Athlete Poliomyelitis Survivors
}

\author{
Shaghayegh Sayyar ${ }^{*}$, Hasan Daneshmandi ${ }^{1}$, Fateme Ebrahimi ${ }^{1}$
}

1. Department of Sport Injuries and Corrective Exercises, Faculty of Physical Education, University of Guilan, Rasht, Iran.

\begin{tabular}{|c|c|}
\hline $\begin{array}{l}\text { Use vour device to scan } \\
\text { and read the article online }\end{array}$ & \\
\hline 口F期回 & Citation: Sayyar Sh, Daneshmandi H, Ebrahimi F. Comparing Symptoms of Post-Polio Syndrome in Athlete and Non-athlete \\
\hline 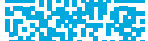 & Poliomyelitis Survivors. Physical Treatments. 2017; 7(1):35-40. https://doi.org/10.29252/NRIP.PTJ.7.1.35 \\
\hline Patsis & dof': https://doi.org/10.29252/NRIP.PTJ.7.1.35 \\
\hline
\end{tabular}

Article info:

Received: 09 Sep. 2016

Accepted: 30 Dec. 2016

Keywords:

Post-Polio Syndrome,

Poliomyelitis, Pain,

Fatigue, Physical health,

Mental health

\begin{abstract}
A B S T RA C T
Purpose: Long term effects of poliomyelitis are known in many countries. Despite no definite label for these signs and symptoms, there is a remarkable similarity among patients with regard to description of the problems and complications of the disease. In this study, we compared the prevalence of symptoms of Post-Polio Syndrome (PPS) in athlete and non-athlete poliomyelitis survivors.

Methods: This is a causal-comparative study. The statistical population consisted of all people who had polio in Kerman Province. Of them, 28 people ( 9 males and 19 females) with the mean(SD) age of 36.61(8.23) years were purposefully selected as the study samples and were grouped in athlete and non-athlete samples. The athlete group comprised 15 people with an mean(SD) age of 34.62(9.42) years who were engaged in physical activities, including swimming, table tennis and sitting volleyball, 3 times a week (on average) during the last year. The non-athlete group also consisted of 15 people with an mean(SD) age of 6.91(38.33) years who didn't do any regular physical activity during this time. With respect to the major indexes of PPS, the symptoms of this syndrome were examined using quality of life questionnaire (SF-36), Fatigue Severity Scale (FSS), and McGill pain questionnaire to evaluate mental and physical function, fatigue and muscular pain, respectively in the study groups. The obtained data were analyzed using descriptive statistics, the Pearson correlation coefficient and Independent $t$ test with SPSS V-22. The significance level was set at $\mathrm{P} \leq 0.05$.
\end{abstract}

Results: The results indicate a high prevalence rate of PPS syndrome including main symptoms of pain and fatigue $(57.53 \%)$. Results also support a significant difference between the physical health of athlete and non-athlete groups $(\mathrm{P}=0.02)$, while no significant difference was observed between these two groups with respect to their mental health status. Also, there was a significant difference between the athlete and non-athlete people in terms of the fatigue $(\mathrm{P}=0.04)$ and pain intensity $(\mathrm{P}=0.04)$ Moreover, there was a significant relationship between the age at the first attack of polio and fatigue $(\mathrm{r}=0.59)$ and physical health $(\mathrm{r}=0.69)$, while there was no significant relationship between the age at the first attack of the disease and pain $(\mathrm{r}=0.24)$ and mental health $(\mathrm{r}=-0.09)$.

Conclusion: Regular physical activity can help patients with a history of polio achieve higher level of health and lower level of functional impairments. Improving the physical condition of these patients through training programs can play an important role in improving their quality of life.

\footnotetext{
* Corresponding Author:

Shaghayegh Sayyar, MSc.

Address: Department of Sport Injuries and Corrective Exercises, Faculty of Physical Education, University of Guilan, Rasht, Iran.

Phone: +98 (911) 9362575

E-mail:shaghayegh.sayyar@yahoo.com
} 


\section{Introduction}

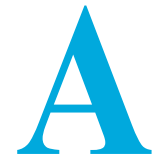

bout 50 million people around the world have been affected by poliomyelitis [1-4]. This is a disease which affects the anterior motor neurons of spinal cord. Of the patients who had polio, a significant number develop new symptoms known as Post-Polio Syndrome (PPS) many years after recovery from the initial attack $[2,5]$. The people with a history of poliomyelitis do not equally suffer from PPS. By emerging the symptoms of PPS, the victims experience some functional changes and musculoskeletal disorders which can be different from the disorders during the polio attack $[1,6]$. In almost $25 \%$ to $50 \%$ of the people, PPS returns after treatment. On average, PPS emerges 3035 years after poliomyelitis, however, this time may varied from 8 to 71 years [7, 8]. Muscular weakness4, muscular atrophy5 and pain in the muscles and joints with fatigue are the main symptoms of PPS $[7,8]$.

Dysphasia, dysphonia and respiratory disorders are the other symptoms of PPS. About $18 \%$ to $29 \%$ of poliomyelitis survivors face with these problems in their daily life [9]. These symptoms can lead to the significant and negative changes in excitability, amount of tumbling and the ability to perform daily activities $[2,10]$. The exact reason for emerging PPS symptoms has not been found yet. Nevertheless, some hypothesis are as follows: reactivation of remained hidden and inactive polio virus or the viral components due to unknown mechanisms, disorder in body's defense mechanisms and degeneration of the spinal cord, neuromuscular changes in the patients with polio due to premature-aging and an extra functionality of the motor neuron which finally lead to the nerve dysfunction.

Body weight-bearing muscles such as leg muscles are involved in this disease more than the other muscles [7]. Generally, PPS is not a life-treating syndrome $[1,7]$. The process of emerging the symptoms after polio is totally benign. The symptoms range from mild weakness to severe weakness, depression, social isolation and dysfunction [7]. According to the evaluations, several comprehensive research studies have been conducted in the developed countries in terms of the disease outbreak and the assessment of secondary symptoms of polio. Moreover, some studies were conducted on the significance of pain, the health quality in these patients' life, strength exercises, endurance exercises, especially dynamic exercises into warm water, the muscular effects on this syndrome, the impact of disability, and the quality of life on the people suffering from the post-polio side effects. The evidence shows that physical exercises have positive effects on the weak and painful muscular groups involved in the disease $[8,10-12]$. However, the people ability should regularly be evaluated and they must be prevented from doing hard and exhausting activities higher than their individual functional level [12].

There is not a standard diagnostic test for PPS and most of the health care specialists are not familiar with the longterm side effects of the polio. Moreover, most patients have difficulties in accepting the disease and their functional decline. Finally, this disability can limit the individual activities at times when lead to more weakness, fatigue and decreased functional activities [8]. The prevalence of PPS in the people with a history of polio still needs more studies. Therefore, with respect to the importance of an active life in people with PPS and improvement of their functional capacities, we conducted a comparative study on the PPS symptoms in the people who had polio (athletes or non-athletes) and the relationships between the disease symptoms and disease duration in them.

\section{Materials and Methods}

This causal-comparative study was conducted from July 2014 to October 2015. The statistical population consisted of all people with a history of polio in Kerman Province. With respect to the limitations on selection of the subjects, 28 people ( 9 males and 19 females) with a history of polio and the mean(SD) age of 36.61(8.23) years were purposefully selected as the study samples and were divided into athlete and non-athlete groups. The athlete group consisted of 15 people with a mean(SD) age of 34.62(9.42) years who were practicing the physical activities, including swimming, table tennis and sitting volleyball, 3 times a week (on average) during the last year. The non-athlete group consisted of 15 people with a mean(SD) age of 6.91(38.33) years who didn't do regular physical activity during this time. In order to observe the principles of research ethics, the study was approved by the Welfare Organization of Kerman Province and a signed written informed consent was obtained from all subjects. The samples had a history of poliomyelitis and aged 20-50 years. The exclusion criterion were the failure to cooperate with the researcher or declining to continue the research.

With respect to the major indexes of PPS, its symptoms were examined by assessing mental and physical function, fatigue and muscular pain in two study groups. In this regard, the Fatigue Severity Scale (FSS) was used to measure the fatigue in these people. The questionnaire consists of 9 questions and scored on a 5-point Likert-type scale. To calculate the total score of the questionnaire, the sum of the points for each question 
was obtained and a higher total score indicates the more fatigue severity of the respondent. Total points are varied between 9 and 81. Thus, the scores 9-27, 28-54 and 54-81 represent "mild fatigue", "moderate fatigue", and "severe fatigue", respectively $(\mathrm{r}=0.96)$.

McGill Pain Questionnaire (MPQ) was used to assess the pain severity. MPQ consists of three major parts and 28 questions, i.e. a set of expressions for measuring the patients' subjective experience of pain (in terms of emotional and sensory perception and assessment). This questionnaire also determines the pain intensity and its other objective features. In addition, it can measure the clinical pain intensity as statistical data. The total point of the questionnaire is varied between 0 (the absence of pain) and 78 (the maximum level of pain) $(r=0.78)$.

The two scales of physical health and mental health from the SF-36 (Quality of Life Questionnaire), the most famous and the most frequently used tool for life quality measurement, were used to measure physical and mental functions $[2,13]$. Eight subscales, including Physical Functioning (PF), role limitations due to physical health (RP), role limitations due to emotional problems (RE), Energy/Fatigue (EF), Emotional Well-being (EW), Social Functioning (SF), Pain (P) and General Health (GH), which each scale made of 2 to 10 items, were evaluated in these two groups using SF-36. Each question consists of five items, and scores between 0 and 100 (i.e. 0-2550-75-100) have been allocated to each item according to the scoring instructions for the questionnaire. For obtaining 8 subscales, the questions for each scale must be summed and divided by the number of questions. Also, the total scale of physical health can be determined by summing the subscales of PF, RP, P and GH. Also, the total scale of mental health can be computed by adding the subscales of RE, EF, EW, and SF. Using Cronbach $\alpha$ coefficients, the reliability of the questionnaire for each aforementioned scale has been reported as $0.90,0.85$, $0.77,0.84,0.65,0.77,0.83$ and 0.71 , respectively.

To examine the normality of data distribution and report the data analysis in this research, K-S test and descriptive and inferential statistics were used. The Independent $t$ test was used to compare the variables of fatigue, muscular pain, physical health and mental health for the athlete and non-athlete subjects, and in order to determine the relationship between each variable and the age during the primary infection to the disease, the Pearson correlation coefficient test were used. All statistical analyses were performed by SPSS V-22 ( $\mathrm{P} \geq 0.05)$.

\section{Results}

KS test results indicated the normality of variables $(\mathrm{P}>0.05)$. Table 1 presents the demographic information of the study subjects. Table 2 presents the prevalence of pain and fatigue in the two groups of the athlete and nonathlete polio survivors as well as the overall prevalence rate of these symptoms. The information for each PPS symptom in athlete and non-athlete polio survivors are presented in Table 3. Also, this Table shows a significant difference between fatigue, pain and physical health in these groups; while there was no significant difference between two groups' mental health status.

Table 4 presents the information for each PPS symptoms with respect to gender. The results do not show any significant difference between these groups. Table 5 indicates a significant relationship between each PPS symptoms and the people's ages at the time of catching poliomyelitis.

Table 1. Demographic information for the subjects

\begin{tabular}{cccc}
\hline Age (Year) & $\begin{array}{c}\text { Age at the Time of Primary } \\
\text { Disease (Year) }\end{array}$ & Height (cm) & Weight (kg) \\
\hline $8.23 \pm 36.61$ & $1.33 \pm 2.47$ & $5.8 \pm 170.5$ & $9.9 \pm 75.6$ \\
\hline
\end{tabular}

Table 2. Prevalence of pain and fatigue in the two groups of the athlete and non-athlete patients

\begin{tabular}{cccc}
\hline variable & Pain & Fatigue & Pain+Fatigue \\
\hline Athlete group & $53.84 \%$ & $61.53 \%$ & $38.46 \%$ \\
\hline Non-athlete group & $80 \%$ & $86.66 \%$ & $66.66 \%$ \\
Total & $67.85 \%$ & $75 \%$ & $53.57 \%$ \\
\hline
\end{tabular}


Table 3. Information for each PPS symptom in the two groups of athlete and non-athlete patients

\begin{tabular}{cccccc}
\hline \multicolumn{2}{c}{ Variable } & $\begin{array}{c}\text { Fatigue } \\
\text { (FSS) }\end{array}$ & $\begin{array}{c}\text { Pain } \\
\text { (McGill) }\end{array}$ & $\begin{array}{c}\text { Physical Health } \\
\text { (FS-36) }\end{array}$ & $\begin{array}{c}\text { Mental Health } \\
\text { (FS-36) }\end{array}$ \\
\hline Mean \pm St. deviation & $\begin{array}{c}\text { Non-athlete } \\
\text { group } \\
\text { Athlete } \\
\text { group }\end{array}$ & $39.62 \pm 9.26$ & $21.31 \pm 12.8$ & $242.57 \pm 76.62$ & $256.69 \pm 70.15$ \\
& Sig. & 0.04 & $36.27 \pm 24.89$ & $163.16 \pm 90.49$ & $210.06 \pm 79.46$ \\
\hline & & 0.04 & 0.02 & 0.06 \\
\hline
\end{tabular}

Table 4. Information for each PPS symptom in the two groups of males and females

\begin{tabular}{|c|c|c|c|c|c|}
\hline \multicolumn{2}{|c|}{ Variable } & $\begin{array}{l}\text { Fatigue } \\
\text { (FSS) }\end{array}$ & $\begin{array}{l}\text { Pain } \\
\text { (McGill) }\end{array}$ & $\begin{array}{l}\text { Physical Health } \\
\text { (FS-36) }\end{array}$ & $\begin{array}{l}\text { Mental Health } \\
\text { (FS-36) }\end{array}$ \\
\hline \multirow{2}{*}{ Mean $\pm S t$. deviation } & Females & $41.42 \pm 11.87$ & $33.21 \pm 23.17$ & $191.1 \pm 90.1$ & $221.07 \pm 71.46$ \\
\hline & Males & $38.89 \pm 6.23$ & $21.11 \pm 14.3$ & $218.88 \pm 98.8$ & $254.18 \pm 89.43$ \\
\hline Sig. & & 0.55 & 0.16 & 0.46 & 0.30 \\
\hline
\end{tabular}

\section{Discussion}

This study aimed to compare the symptoms of PPS, including muscular pain, fatigue and physical and mental health, between athlete and non-athlete people with a history of polio with respect to their sex and age. The results indicate a high prevalence of PPS syndrome with regard to pain and fatigue $(57.53 \%)$, especially in nonathlete people. Similarly, Talebian et al. reported 55.66\% prevalence for PPS syndrome [8]. In a study on the prevalence of PPS syndrome and its risk factors, Bang et al. reported that on average $61.6 \%$ of the people with a history of polio show the symptoms of PPS and most of them are middle-aged [7]. Ramlow et al. also reported a PPS prevalence of $28.5 \%$ [14].

With respect to the research findings, a significant percentage of the polio survivors $(67.85 \%)$ suffer from annoying or very annoying muscular and arthritis pain after passing so many years. Several years after the initial symptoms, the people with polio suffer from symptoms resulted from the primary infection following a period of stagnation; a large number of samples reported muscular pain and arthritis and most patients experience a renewed weakness in a manner that decreases their ability in doing daily activities [5]. Talebian et al. reported the prevalence rates of $88 \%$ and $72.7 \%$ for muscular and arthritis pains, respectively [8]. In a study on the relationship between the pain intensity and its effects on daily activities of people with PPS, Sheth et al. concluded that $44 \%, 39 \%$, and $37 \%$ of patients suffer from the muscular, arthritis and muscular-arthritis pains, respectively. Most patients suffer from the pain, especially in their knees, shoulders, thighs and waists, when doing activities, which can be relieved by resting. Some people also complain of a prolonged pain during a day. The greatest amount of the pain is around arms, legs, and feet muscles and there is a significant relationship between the pain intensity and a disruption of daily activities [4].

Werhangen et al. also claimed that pain is one of the most common and serious problems of the people with PPS and they reported a prevalence rate of $91 \%$ for it. They stated that $68 \%$ of the people with PPS suffer from

Table 5. Correlation between PPS symptoms and the age at the time of catching poliomyelitis

\begin{tabular}{|c|c|c|}
\hline Variable & Correlation & Sig. \\
\hline Fatigue & -0.59 & 0.001 \\
\hline Pain & -0.24 & 0.05 \\
\hline Physical health & 0.69 & 0.001 \\
\hline Mental health & -0.09 & 0.05 \\
\hline
\end{tabular}


severe pain which can decrease their quality of life [3]. In the highest level, the muscular pain results in sleep disorder as well as a disturbance when exercising physical activities in which many muscular structures are involved [15]. The muscular pains are usually occur at the upper limbs and can significantly affect the individual's function. The physical stresses can intensify pain, while resting and heating can relieve it [16].

The research results indicate a $75 \%$ prevalence rate for the moderate and severe pains among the polio survivors, while Talebian et al. reported an $86 \%$ prevalence rate of fatigue in these people [8]. In an assessment of walking in the people with PPS, Vreed et al. concluded that these people experience more muscular exhaustion and respiratory disorders per a certain physical activity as compared to the healthy people [9]. Fatigue and muscular pain limit the mental and physical functionality of the people with PPS, and this lower functionality in middle-aged people is more prevalent than other age groups [17].

Results showed a significant difference between the physical health of athletes and non-athletes with polio $(\mathrm{P}=0.02)$, while no significant difference was seen between the mental health of these two groups. The athletes with a history of polio enjoy greater physical health [5]. Vreed et al. concluded that the people with PPS experience a lower health level than others, and enhancing these people's physical and emotional state can play an important role in improving their quality of life [9]. Also, in a comparative study on the impact of lifestyle changes with physical exercise or without physical exercise on the fatigue and functional capacity of 18-65 years old people with PPS, Sharma et al observed that doing physical exercises with a change in the lifestyle can significantly improve the fatigue, functional capacity and physical function of the people with PPS [10]. However, in addition to rejecting a sedentary lifestyle, these patients should avoid doing intense physical activities [18]. In this regard, in a study on the physical and mental health of the people with polio and comparing them with healthy population using the SF-36 quality of life questionnaire, Jung et al. found that the physical and mental health of these patients were significantly lower than their healthy peers [19]. The current study results are consistent with the findings of researchers such as Ostlund et al. who concluded that the people with PPS suffer from a lower mental and physical health compared to the other people [20].

Also, the research results indicated a significant difference between the athletes and non-athletes survivors of polio in terms of the fatigue $(\mathrm{P}=0.04)$ and pain intensity
$(\mathrm{P}=0.04)$. However, Ramlow et al. reported that the level of physical activity cannot affect the presentation of PPS symptoms after recovery [17]. According to the literature, people with a history of polio who regularly do physical exercises enjoy more public health and complain less from arthritis, muscular pain, fatigue and functional disorders as compared to the inactive survivors of polio [16].

One of the factors affecting the PPS symptoms is sex, as females are more likely prone to the syndrome than males $[5,17]$. However, according to the current study results, there was no significant difference between males and females in terms of variables studied in the field of PPS, while some evidence shows that the males with PPS enjoy a higher quality of life as compared to the females [9]. This study results are not consistent with the results of Shimoun et al. and Ostlund et al. who indicated a greater prevalence rate of symptoms in the females with polio than the male counterparts [20]. Bang et al. reported the sex, age, history of muscle paralysis, and pain and gait disorders as the potential risk factors for PPS symptoms [5]. Ramlow et al. believed that the people who suffered from a permanent impairment in the first attack of polio and also females are more prone to PPS [17]. According to the results obtained by Jung et al., although catching polio during childhood can decrease the mental and physical health in both males and females, the health level in males is significantly better than female survivors [19].

In addition, the findings of this research indicate a significant relationship between the age at the first attack of polio and variables of fatigue $(\mathrm{r}=0.59)$ and physical health status $(\mathrm{r}=0.69)$, while no significant relationship was seen between the age at the first attack of polio and variables of pain $(\mathrm{r}=0.24)$ and mental health status $(\mathrm{r}=0.09)$. Also, there was no significant relationship between the age and PPS symptoms. Ramlow et al. also reported that the age of patients during the disease and the disease severity do not affect the PPS symptoms [14].

However, the results from this research are not consistent with the findings obtained by the researchers such as Bang et al $[5,7]$. Older age and level of the fatigue and muscular pain may limit the people's mental and physical functionality, and the middle-aged people suffer more functional decline as compared to the other age groups [14]. At the first attacks of polio, younger people are more likely prone to PPS [7]. The results of various studies in this filed indicate that the quality of life of people with a history of polio, especially in terms of physical aspects, may significantly be decreased with increasing age in males and females [3, 4]. 
Future research is suggested on the relationship between fatigue, pain, physical and mental health in PPS. Very few studies have explored exercise as a means to reduce fatigue, pain or pain-related problems in PPS population, so researchers should also attempt to find an effective treatment for symptoms of PPS. Regular physical activity can help patients with a history of poliomyelitis to achieve a higher level of health and lower level of functional impairments. Improving the physical condition of these patients through training programs can play an important role in improving their quality of life.

\section{Acknowledgments}

This research did not receive any specific grant from funding agencies in the public, commercial, or not-forprofit sectors. The authors hereby express their deep sense of gratitude and indebtedness to the authorities of the Welfare Organization of Kerman Province and all of the associates for their valuable guidance, encouragement and co-operation in preparing this paper.

\section{Conflict of Interest}

The authors declared no conflicts of interest.

\section{References}

[1] Bickerstaffe A, Beelen A, Nollet F. Change in physical mobility over 10 years in post-polio syndrome. Neuromuscular Disorders. 2015; 25(3):225-30. doi:10.1016/j.nmd.2014.11.015

[2] Gonzalez H, Olsson T, Borg K. Management of postpolio syndrome. The Lancet Neurology. 2010; 9(6):634-42. doi: 10.1016/s1474-4422(10)70095-8

[3] Werhagen L, Borg K. Impact of pain on quality of life in patients with post polio syndrome. Journal of Rehabilitation Medicine. 2013; 45(2):161-3. doi: 10.2340/16501977-1096

[4] Sheth MS, Ghoghari B, Vyas NJ. Presentation and impact of pain in persons with post polio syndrome: A cross sectional survey study. Disability, CBR \& Inclusive Development. 2014; 24(4). doi: 10.5463/dcid.v24i4.244

[5] Basmajian JV, Wolf SL. Therapeutic exercise. Oxford: Pergamon Press; 1992.

[6] Brogårdh C, Lexell J, Lundgren Nilsson Å. Construct validity of a new rating scale for self reported impairments in persons with late effects of polio. PM\&R. 2013; 5(3):176-81. doi: 10.1016/j.pmrj.2012.07.007

[7] Bang H, Suh JH, Lee SY, Kim K, Yang EJ, Jung SH, et al. Post polio syndrome and risk factors in korean polio survivors: A baseline survey by telephone interview. Annals of Rehabilitation Medicine. 2014; 38(5):637. doi: 10.5535/ arm.2014.38.5.637

[8] Talebian S, Olyaei GR. [Survey of post polio syndrome in Tehran (Persian)]. Tehran University Medical Journal. 2009; 67(1):70-75.

[9] Reede K, Henriksson J, Borg K, Henriksson M. Gait characteristics and influence of fatigue during the 6-minute walk test in patients with post polio syndrome. Journal of Rehabilitation Medicine. 2013; 45(9):924-8. doi: 10.2340/165019771209

[10] Sharma SS, Sheth MS, Vyas NJ. Fatigue and functional capacity in persons with post polio syndrome: Short term effects of exercise and lifestyle modification compared to lifestyle modification alone. Disability, CBR \& Inclusive Development. 2014; 25(3):78. doi: 10.5463/dcid.v25i3.335

[11] Jensen MP, Alschuler KN, Smith AE, Verrall AM, Goetz MC, Molton IR. Pain and fatigue in persons with postpolio syndrome: Independent effects on functioning. Archives of Physical Medicine and Rehabilitation. 2011; 92(11):1796-801. doi: 10.1016/j.apmr.2011.06.019

[12] Stoelb BL, Carter GT, Abresch RT, Purekal S, McDonald $\mathrm{CM}$, Jensen MP. Pain in persons with postpolio syndrome: Frequency, intensity, and impact. Archives of Physical Medicine and Rehabilitation. 2008; 89(10):1933-40. doi: 10.1016/j. apmr.2008.03.018

[13] Demir CF, Berilgen MS, Mungen B, Bulut S. Do polio survivors have a higher risk of epilepsy. Epilepsy Research. 2012 98(1):72-5. doi: 10.1016/j.eplepsyres.2011.08.019

[14] Ramlow J, Alexander M, LaPorte R, Kaufmann C, Kuller L. Epidemiology of the post polio syndrome. American Journal of Epidemiology. 1992; 136(7):769-86. doi: 10.1093/ aje/136.7.769

[15] Boonyapisit K. Poliomyelitis and post poliomyelitis syndrome. Neuromuscular Disorders in Clinical Practice. 2013, 383-93. doi: 10.1007/978-1-4614-6567-6_19

[16] Winberg C, Flansbjer UB, Carlsson G, Rimmer J, Lexell J. Physical activity in persons with late effects of polio: A descriptive study. Disability and Health Journal. 2014; 7(3):3028. doi: 10.1016/j.dhjo.2014.02.003

[17] Arzu MD. The role of EMG investigations in Post-polio Syndrome. Journal of Rehabilitation Medicine. 2014; 46:568-608.

[18] Widar M, Ahlstrom G. Experiences and consequences of pain in persons with post polio syndrome. Journal of Advanced Nursing. 1998; 28(3):606-13. doi: 10.1046/j.1365 2648.1998.00695.x

[19] Jung TD, Broman L, Stibrant Sunnerhagen K, Gonzalez H, Borg K. Quality of life in Swedish patients with post polio syndrome with a focus on age and sex. International Journal of Rehabilitation Research. 2014; 37(2):173-9. doi: 10.1097/ mrr.0000000000000052

[20] Östlund G, Wahlin ̊̊, Sunnerhagen KS, Borg K. Post polio syndrome: Fatigued patients a specific subgroup. Journal of Rehabilitation Medicine. 2011; 43(1):39-45. doi: $10.2340 / 16501977-0634$ 